\title{
Universal One-Way Hash Functions via Inaccessible Entropy
}

\author{
Iftach Haitner ${ }^{1}$, Thomas Holenstein ${ }^{2}$, Omer Reingold ${ }^{3, \star}$, \\ Salil Vadhan ${ }^{4, \star \star}$, and Hoeteck Wee ${ }^{5, \star \star \star}$ \\ 1 Microsoft Research, New England \\ iftach@microsoft.com \\ 2 Department of Computer Science, ETH Zurich \\ thomas.holenstein@inf .ethz.ch \\ 3 Microsoft Research, Silicon Valley and Weizmann Institute of Science \\ omreing@microsoft.com \\ ${ }^{4}$ School of Engineering \& Applied Sci. and Center for Research on Computation \& Society, \\ Harvard University \\ salil@seas.harvard.edu \\ ${ }^{5}$ Queens College, CUNY \\ hoeteck@cs . qc . cuny . edu
}

\begin{abstract}
This paper revisits the construction of Universal One-Way Hash Functions (UOWHFs) from any one-way function due to Rompel (STOC 1990). We give a simpler construction of UOWHFs, which also obtains better efficiency and security. The construction exploits a strong connection to the recently introduced notion of inaccessible entropy (Haitner et al. STOC 2009). With this perspective, we observe that a small tweak of any one-way function $f$ is already a weak form of a UOWHF: Consider $F(x, i)$ that outputs the $i$-bit long prefix of $f(x)$. If $F$ were a UOWHF then given a random $x$ and $i$ it would be hard to come up with $x^{\prime} \neq x$ such that $F(x, i)=F\left(x^{\prime}, i\right)$. While this may not be the case, we show (rather easily) that it is hard to sample $x^{\prime}$ with almost full entropy among all the possible such values of $x^{\prime}$. The rest of our construction simply amplifies and exploits this basic property.

With this and other recent works, we have that the constructions of three fundamental cryptographic primitives (Pseudorandom Generators, Statistically Hiding Commitments and UOWHFs) out of one-way functions are to a large extent unified. In particular, all three constructions rely on and manipulate computational notions of entropy in similar ways. Pseudorandom Generators rely on the well-established notion of pseudoentropy, whereas Statistically Hiding Commitments and UOWHFs rely on the newer notion of inaccessible entropy.
\end{abstract}

Keywords: computational complexity, cryptography, hashing, target collisionresistance, one-way functions.

\footnotetext{
* Supported by US-Israel BSF grant 2006060.

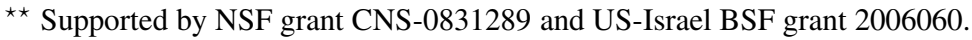

$\star \star \star$ Supported in part by PSC-CUNY Award \#6014939 40.
} 


\section{Introduction}

Universal one-way hash functions (UOWHFs), as introduced by Naor and Yung [10], are a weaker form of collision-resistant hash functions. The standard notion of collision resistance requires that given a randomly chosen function $f \stackrel{R}{\leftarrow} \mathcal{F}$ from the hash family, it is infeasible to find any pair of distinct inputs $x, x^{\prime}$ such that $f(x)=f\left(x^{\prime}\right)$. UOWHFs only require target collision resistance, where the adversary must specify one of the inputs $x$ before seeing the description of the function $f$. Formally:

Definition 1. A family of functions $\mathcal{F}_{k}=\left\{F_{z}:\{0,1\}^{n(k)} \rightarrow\{0,1\}^{m(k)}\right\}_{z \in\{0,1\}^{k}}$ is a family of universal one-way hash functions (UOWHFs) if it satisfies:

1. Efficiency: Given $z \in\{0,1\}^{k}$ and $x \in\{0,1\}^{n(k)}, F_{z}(x)$ can be evaluated in time $\operatorname{poly}(n(k), k)$.

2. Shrinking: $m(k)<n(k)$.

3. Target Collision Resistance: For every probabilistic polynomial-time adversary A, the probability that $A$ succeeds in the following game is negligible in $k$ :

(a) Let $(x$, state $) \leftarrow A\left(1^{k}\right) \in\{0,1\}^{n(k)} \times\{0,1\}^{*}$.

(b) Choose $z \stackrel{R}{\leftarrow}\{0,1\}^{k}$.

(c) Let $x^{\prime} \stackrel{R}{\leftarrow} A($ state,$z) \in\{0,1\}^{n(k)}$.

(d) $A$ succeeds if $x \neq x^{\prime}$ and $F_{z}(x)=F_{z}\left(x^{\prime}\right)$.

It turns out that this weaker security property suffices for many applications. The most immediate application given in [10] is secure fingerprinting, whereby the pair $(f, f(x))$ can taken as a compact "fingerprint" of a large file $x$, such that it is infeasible for an adversary, seeing the fingerprint, to change the file $x$ to $x^{\prime}$ without being detected. More dramatically, Naor and Yung [10] also showed that UOWHFs can be used to construct secure digital signature schemes, whereas all previous constructions (with proofs of security in the standard model) were based on trapdoor functions (as might have been expected to be necessary due to the public-key nature of signature schemes). More recently, UOWHFs have been used in the Cramer-Shoup encryption scheme [3] and in the construction of statistically hiding commitment schemes from one-way functions [4, 5].

Naor and Yung [10] gave a simple and elegant construction of UOWHFs from any one-way permutation. Subsequently, Rompel [11] gave a much more involved construction to prove that UOWHFs can be constructed from an arbitrary one-way function, thereby resolving the complexity of UOWHFs (as one-way functions are the minimal complexity assumption for complexity-based cryptography, and are easily implied by UOWHFs) 1 While complications may be expected for constructions from arbitrary one-way functions (due to their lack of structure), Rompel's analysis also feels quite ad hoc. In contrast, the construction of pseudorandom generators from oneway functions of [7], while also somewhat complex, involves natural abstractions (e.g., pseudoentropy) that allow for modularity and measure for what is being achieved at each stage of the construction.

In this paper, we give simpler constructions of UOWHFs from one-way functions, based on (a variant of) the recently introduced notion of inaccessible entropy [5]. In addition, one of the constructions obtains slightly better efficiency and security.

\footnotetext{
${ }^{1}$ More details of Rompel's proof are worked out, with some corrections, in [12, 9].
} 


\subsection{Inaccessible Entropy}

For describing our construction, it will be cleaner to work with a variant of UOWHFs where there is a single shrinking function $F:\{0,1\}^{n} \rightarrow\{0,1\}^{m}$ (for each setting of the security parameter $k$ ) such that it is infeasible to find collisions with random inputs. That is, an adversary $A$ is given a uniformly random $x \stackrel{\mathrm{R}}{\longleftarrow}\{0,1\}^{n}$, outputs an $x^{\prime}$ such that $F\left(x^{\prime}\right)=F(x)$, and succeeds if $x^{\prime} \neq x$ Note that we can assume without loss of generality that $x^{\prime}=A(x)$ is always a preimage of $F(x)$ ( $A$ has the option of outputting $x$ in case it does not find a different preimage); we refer to an algorithm $A$ with this property as an $F$-collision finder.

Our construction is based on an entropy-theoretic view of UOWHFs. The fact that $F$ is shrinking implies that there are many preimages $x^{\prime}$ available to $A$. Indeed, if we consider an (inefficient) adversary $A(x)$ that outputs $x^{\prime} \stackrel{\mathrm{R}}{\leftarrow} F^{-1}(F(x))$ and let $X$ be a random variable uniformly distributed on $\{0,1\}^{n}$, then

$$
\mathrm{H}(A(X) \mid X)=\mathrm{H}(X \mid F(X)) \geq n-m,
$$

where $\mathrm{H}(\cdot \mid \cdot)$ denotes conditional Shannon entropy. (See Section 2 for more definitional details.) We refer to the quantity $\mathrm{H}(X \mid F(X))$ as the real entropy of $F^{-1}$.

On the other hand, the target collision resistance means that effectively only one of the preimages is accessible to $A$. That is for every probabilistic polynomial-time $F$ collision finder $A$, we have $\operatorname{Pr}[A(X) \neq X]=\operatorname{neg}(n)$, which is equivalent to requiring that:

$$
\mathrm{H}(A(X) \mid X)=\operatorname{neg}(n)
$$

for all probabilistic polynomial-time $F$-collision finders $A$. (If $A$ can find a collision $X^{\prime}$ with nonnegligible probability, then it can achieve nonnnegligible conditional entropy by outputting $X^{\prime}$ with probability $1 / 2$ and outputting $X$ with probability $1 / 2$.) We refer to the maximum of $\mathrm{H}(A(X) \mid X)$ over all efficient $F$-collision finders as the accessible entropy of $F^{-1}$. We stress that accessible entropy refers to an upper bound on a form of computational entropy, in contrast to the Håstad et al.'s notion of pseudoentropy [7].

Thus, a natural weakening of the UOWHF property is to simply require a noticeable gap between the real and accessible entropies of $F^{-1}$. That is, for every probabilistic polynomial-time $F$-collision finder $A$, we have $\mathrm{H}(A(X) \mid X)<\mathrm{H}(X \mid F(X))-\Delta$, for some noticeable $\Delta$, which we refer to as the inaccessible entropy of $F$.

\subsection{Our Constructions}

Our constructions of UOWHFs have two parts. First, we show how to obtain a function with noticeable inaccessible entropy from any one-way function. Second, we show how to build a UOWHF from any function with inaccessible entropy.

OWFs $\Rightarrow$ Inaccessible Entropy. Given a one-way function $f:\{0,1\}^{n} \rightarrow\{0,1\}^{m}$, we show that a random truncation of $f$ has inaccessible entropy. Specifically, we define $F(x, i)$ to be the first $i$ bits of $f(x)$.

\footnotetext{
${ }^{2}$ It is easy to convert any such function $F$ into a standard UOWHF family by defining $F_{z}(x)=$ $F(z+x)$.
} 
To see that this works, suppose for contradiction that $F$ does not have noticeable inaccessible entropy. That is, we have an efficient adversary $A$ that on input $(x, i)$ can sample from the set $S(x, i)=\left\{x^{\prime}: f\left(x^{\prime}\right)_{1 \ldots i}=f(x)_{1 \ldots i}\right\}$ with almostmaximal entropy, which is equivalent to sampling according to a distribution that is statistically close to the uniform distribution on $S(x, i)$. We can now use $A$ to construct an inverter $\operatorname{In} v$ for $f$ that works as follows on input $y$ : choose $x_{0} \stackrel{R}{\leftarrow}\{0,1\}^{n}$, and then for $i=1, \ldots, n$ generate a random $x_{i} \stackrel{\mathrm{R}}{\leftarrow} A\left(x_{i-1}, i-1\right)$ subject to the constraint that $f\left(x_{i}\right)_{1, \cdots, i}=y_{1, \cdots, i}$. The latter step is feasible, since we are guaranteed that $f\left(x_{i}\right)_{1, \ldots, i-1}=y_{1, \cdots, i-1}$ by the fact that $A$ is an $F$-collision finder, and the expected number of trials needed get agreement with $y_{i}$ is at most 2 (since $y_{i} \in\{0,1\}$, and $y$ and $f\left(x_{i}\right)$ are statistically close). It is not difficult to show that when run on andom output $Y$ of $f$, Inv produces an almost-uniform preimage of $Y$. This contradicts the one-wayness of $f$. Indeed, we only need $f$ to be a distributional one-way function [8], whereby it is infeasible to generate almost-uniform preimages under $f$.

Inaccessible Entropy $\Rightarrow$ UOWHFs. Once we have a non-negligible amount of inaccessible entropy, we can construct a UOWHF via a series of standard transformations.

1. Repetition: By evaluating $F$ on many inputs, we can increase the amount of inaccessible entropy from $1 / \operatorname{poly}(n)$ to $\operatorname{poly}(n)$. Specifically, we take $F^{t}\left(x_{1}, \ldots, x_{t}\right)=\left(F\left(x_{1}\right), \ldots, F\left(x_{t}\right)\right)$ where $t=\operatorname{poly}(n)$. This transformation also has the useful effect of converting the real entropy of $F^{-1}$ to min-entropy.

2. Hashing Inputs: By hashing the input to $F$ (namely taking $F^{\prime}(x, g)=$ $(F(x), g(x))$ for a universal hash function $g$ ), we can reduce both the real (min)entropy and the accessible entropy so that $\left(F^{\prime}\right)^{-1}$ still has a significant amount of real entropy, but has (weak) target collision resistance (on random inputs).

3. Hashing Outputs: By hashing the output to $F$ (namely taking $F^{\prime}(x, g)=$ $g(F(x))$ ), we can reduce the output length of $F$ to obtain a shrinking function that still has (weak) target collision resistance.

There are two technicalities that occur in the above steps. First, hashing the inputs only yields weak target collision resistance; this is due to the fact that accessible Shannon entropy is an average-case measure and thus allows for the possibility that the adversary can achieve high accessible entropy most of the time. Fortunately, this weak form of target collision resistance can be amplified to full target collision resistance using another application of repetition and hashing (similar to [1]).

Second, the hashing steps require having a fairly accurate estimate of the real entropy. This can be handled similarly to [7, 11], by trying all (polynomially many) possibilities and concatenating the resulting UOWHFs, at least one of which will be target collision resistant.

A More Efficient Construction. We obtain a more efficient construction of UOWHFs by hashing the output of the one-way function $f$ before truncating. That is, we define $F(x, g, i)=\left(g, g(f(x))_{1 \cdots i}\right)$. This function is in the spirit of the function that Rompel [11] uses as a first step, but our function uses three-wise independent hash 
function instead of $n$-wise independent one, and enjoys a much simpler structure 3 Our analysis of this function is significantly simpler than Rompel's and can be viewed as providing a clean abstraction of what it achieves (namely, inaccessible entropy) that makes the subsequent transformation to a UOWHF much easier.

We obtain improved UOWHF parameters over our first construction for two reasons. First, we obtain a larger amount of inaccessible entropy: $(\log n) / n$ bits instead of roughly $1 / n^{4}$ bits. Second, we obtain a bound on a stronger form of accessible entropy, which enables us to get full target collision resistance when we hash the inputs, avoiding the second amplification step.

This construction yields better parameters than Rompel's original construction. A one-way function of input length $n$ yields a UOWHF with output length $\tilde{O}\left(n^{7}\right)$, improving Rompel's bound of $\tilde{O}\left(n^{8}\right)$. Additionally, we are able to reduce the key length needed: Rompel's original construction uses a key of length $\tilde{O}\left(n^{12}\right)$, whereas our construction only needs a key of length $\tilde{O}\left(n^{7}\right)$. If we allow the construction to utilize some nonuniform information (namely an estimate of the real entropy of $F^{-1}$ ), then we obtain output length $\tilde{O}\left(n^{5}\right)$, improving Rompel's bound of $\tilde{O}\left(n^{6}\right)$. For the key length, the improvement in this case is from $\tilde{O}\left(n^{7}\right)$ to $\tilde{O}\left(n^{5}\right)$. Of course, these bounds are still far from practical, but they illustrate the utility of inaccessible entropy in reasoning about UOWHFs, which may prove useful in future constructions (whether based on one-way functions or other building blocks).

\subsection{Perspective}

The idea of inaccessible entropy was introduced in [5] for the purpose of constructing statistically hiding commitment schemes from one-way functions and from zeroknowledge proofs. There, the nature of statistically hiding commitments necessitated more involved notions of inaccessible entropy than we present here - inaccessible entropy was defined in [5] for interactive protocols and for "generators" that output many blocks, where one considers adversaries that try to generate next-messages or next-blocks of high entropy. In such a setting, it is necessary to have the adversary privately "justify" that it is behaving consistently with the honest party, and to appropriately discount the entropy in case the adversary outputs an invalid justification.

Here, we are able to work with a much simpler form of inaccessible entropy. The simplicity comes from the noninteractive nature of UOWHFs (so we only need to measure the entropy of a single string output by the adversary), and the fact that we can assume without loss of generality that the adversary behaves consistently with the honest party. Thus, the definitions here can serve as a gentler introduction to the concept of inaccessible entropy. On the other hand, the many-round notions from [5] allow for a useful "entropy equalization" transformation that avoids the need to try all possible guesses for the entropy. We do not know an analogous transformation for constructing UOWHFs. We also note that our simple construction of a function with inaccessible entropy by randomly truncating a one-way function (and its analysis) is inspired by the the construction of an "inaccessible entropy generator" from a one-way function in [5].

\footnotetext{
${ }^{3}$ Rompel started with the function $f^{\prime}\left(z, g_{1}, g_{2}\right):=\left(g_{2}\left(f_{0}\left(g_{1}(z)\right)\right), g_{1}, g_{2}\right)$, where $g_{1}$ and $g_{2}$ are $n$-wise independent hash-functions, and $f_{0}$ is defined as $f_{0}(x, y, i)=\left(f(x), y^{n-i}, 0^{i}\right)$.
} 
Finally, with our constructions, the proof that one-way functions imply UOWHFs now parallels those of pseudorandom generators [7, 6] and statistically hiding commitments [4, 5], with UOWHFs and statistically hiding commitments using dual notions of entropy (high real entropy, low accessible entropy) to pseudorandom generators (low real entropy, high pseudoentropy).

\section{Inaccessible Entropy for Inversion Problems}

We will refer to several measures of entropy in this work (proofs are omitted due to the lack of space). For a random variable $X$ and $x \in \operatorname{Supp}(X)$, we define the sampleentropy of $x$ with respect to $X$ to be the quantity

$$
\mathrm{H}_{X}(x):=\log (1 / \operatorname{Pr}[X=x]) .
$$

Using this notion, we can define the Shannon entropy $\mathrm{H}(X)$ and min-entropy $\mathrm{H}_{\infty}(X)$ as follows:

$$
\mathrm{H}(X):=\mathrm{E}_{x \leftarrow X}\left[\mathrm{H}_{X}(x)\right] \quad \text { and } \quad \mathrm{H}_{\infty}(X):=\min _{x \in \operatorname{Supp}(X)} \mathrm{H}_{X}(x)
$$

We will also discuss the max-entropy $\mathrm{H}_{0}(X):=\log (1 /|\operatorname{Supp}(X)|)$. It can be shown that $\mathrm{H}_{\infty}(X) \leq \mathrm{H}(X) \leq \mathrm{H}_{0}(X)$ with equality if and only if $X$ is flat.

As discussed in the introduction, for a function $F$, we define the real entropy of $F^{-1}$ to be the amount of entropy left in the input after revealing the output.

Definition 2. Let $n$ be a security parameter, and $F:\{0,1\}^{n} \rightarrow\{0,1\}^{m}$ a function. We say that $F^{-1}$ has real Shannon entropy $k$ if

$$
\mathrm{H}(X \mid F(X))=k,
$$

where $X$ is uniformly distributed on $\{0,1\}^{n}$. We say that $F^{-1}$ has real min-entropy at least $k$ if there is a negligible function $\varepsilon=\varepsilon(n)$ such that

$$
\operatorname{Pr}_{x \stackrel{R}{\leftarrow} X}\left[\mathrm{H}_{X \mid F(X)}(x \mid F(x)) \geq k\right] \geq 1-\varepsilon(n) .
$$

We say that $F^{-1}$ has real max-entropy at most $k$ if there is a negligible function $\varepsilon=$ $\varepsilon(n)$ such that

$$
\operatorname{Pr}_{x \leftarrow X}\left[\mathrm{H}_{X \mid F(X)}(x \mid F(x)) \leq k\right] \geq 1-\varepsilon(n) .
$$

Note that more concrete formulas for the entropies above are:

$$
\mathrm{H}_{X \mid F(X)}(x \mid F(x))=\log \left|F^{-1}(F(x))\right| \text { and } \mathrm{H}(X \mid F(X))=\mathrm{E}\left[\log \left|F^{-1}(F(X))\right|\right] .
$$

As our goal is to construct UOWHFs that are shrinking, achieving high real entropy is a natural intermediate step. Indeed, the amount by which $F$ shrinks is a lower bound on the real entropy of $F^{-1}$ :

Proposition 1. If $F:\{0,1\}^{n} \rightarrow\{0,1\}^{m}$, then the real Shannon entropy of $F^{-1}$ is at least $n-m$, and the real min-entropy of $F^{-1}$ is at least $n-m-s$ for any $s=\omega(\log n)$. 
To motivate the definition of accessible entropy, we now present an alternative formulation of real entropy in terms of the entropy that computationally unbounded "collision-finding" adversaries can generate.

Definition 3. For a function $F:\{0,1\}^{n} \rightarrow\{0,1\}^{m}$, an $F$-collision-finder is a randomized algorithm $A$ such that for every $x \in\{0,1\}^{n}$ and coin tosses $r$ for $A$, we have $A(x ; r) \in F^{-1}(F(x))$.

Note that $A$ is required to always produce an input $x^{\prime} \in\{0,1\}^{n}$ such that $F(x)=$ $F\left(x^{\prime}\right)$. This is a reasonable constraint because $A$ has the option of outputting $x^{\prime}=x$ if it does not find true collision. We consider $A$ 's goal to be maximizing the entropy of its output $x^{\prime}=A(x)$, given a random input $x$. If we let $A$ be computationally unbounded, then the optimum turns out to equal exactly the real entropy:

Proposition 2. Let $F:\{0,1\}^{n} \rightarrow\{0,1\}^{m}$. Then the real Shannon entropy of $F^{-1}$ equals the maximum of $\mathrm{H}(A(X ; R) \mid X)$ over all (computationally unbounded) $F$ collision finders $A$, where random variable $X$ is uniformly distributed in $\{0,1\}^{n}$ and $R$ is uniformly random coin tosses for $A$. That is,

$$
\mathrm{H}(X \mid F(X))=\max _{A} \mathrm{H}(A(X ; R) \mid X),
$$

where the maximum is taken over all F-collision finders $A$.

The notion of accessible entropy simply restricts the above to efficient adversaries, e.g. those that run in probabilistic polynomial time (PPT for short):

Definition 4. Let $n$ be a security parameter and $F:\{0,1\}^{n} \rightarrow\{0,1\}^{m}$ a function. We say that $F^{-1}$ has accessible Shannon entropy at most $k$ if for every PPT $F$-collisionfinder $A$, we have

$$
\mathrm{H}(A(X ; R) \mid X) \leq k
$$

for all sufficiently large $n$, where random variable $X$ is uniformly distributed on $\{0,1\}^{n}$ and $R$ is uniformly random coin tosses for $A$.

As usual, it is often useful to have an upper bound not only on Shannon entropy, but on the max-entropy (up to some negligible statistical distance). Recall that a random variable $Z$ has max-entropy at most $k$ iff the support of $Z$ is contained in a set of size $2^{k}$. Thus, we require that $A(X ; R)$ is contained in a set $L(X)$ of size at most $2^{k}$, except with negligible probability:

Definition 5. Let $n$ be a security parameter and $F:\{0,1\}^{n} \rightarrow\{0,1\}^{m}$ a function. For $p=p(n) \in[0,1]$, we say that $F^{-1}$ has $p$-accessible max-entropy at most $k$ if for every PPT $F$-collision-finder A, there exists a family of sets $\{L(x)\}_{x \in \operatorname{Supp}(X)}$ each of size at most $2^{k}$ such that $x \in L(x)$ for all $x \in \operatorname{Supp}(X)$ and

$$
\operatorname{Pr}[A(X ; R) \in L(X)] \geq 1-p
$$

for all sufficiently large $n$, where random variable $X$ is uniformly distributed on $\{0,1\}^{n}$ and $R$ is uniformly random coin tosses for $A$. In addition, if $p=\varepsilon(n)$ for some negligible function $\varepsilon(\cdot)$, then we simply say that $F^{-1}$ has accessible max-entropy at most $k$. 
The reason that having an upper bound on accessible entropy is useful as an intermediate step towards constructing UOWHFs is that accessible max-entropy 0 is equivalent to target collision resistance (on random inputs):

Definition 6. Let $F:\{0,1\}^{n} \rightarrow\{0,1\}^{m}$ be a function. For $q=q(n) \in[0,1]$, we say that $F$ is $q$-collision-resistant on random inputs if for every PPT $F$-collision-finder $A$,

$$
\operatorname{Pr}[A(X ; R)=X] \geq q,
$$

for all sufficiently large $n$, where random variable $X$ is uniformly distributed on $\{0,1\}^{n}$ and $R$ is uniformly random coin tosses for $A$. In addition, if $q=1-\varepsilon(n)$ for some negligible function $\varepsilon(\cdot)$, we say that $F$ is collision-resistant on random inputs.

Lemma 1. Let $n$ be a security parameter and $F:\{0,1\}^{n} \rightarrow\{0,1\}^{m}$ be a function. Then, for any $p=p(n) \in(0,1)$, the following statements are equivalent:

(1) $F^{-1}$ has $p$-accessible max-entropy 0 .

(2) $F$ is $(1-p)$-collision-resistant on random inputs.

In particular, $F^{-1}$ has accessible max-entropy 0 iff $F$ is collision-resistant on random inputs.

While bounding $p$-accessible max-entropy with negligible $p$ is our ultimate goal, one of our constructions will work by first giving a bound on accessible Shannon entropy, and then deducing a bound $p$-accessible max-entropy for a value of $p<1$ using the following lemma:

Lemma 2. Let $n$ be a security parameter and $F:\{0,1\}^{n} \rightarrow\{0,1\}^{m}$ be a function. If $F^{-1}$ has accessible Shannon entropy at most $k$, then $F^{-1}$ has p-accessible max-entropy at most $k / p+O\left(2^{-k / p}\right)$ for any $p=p(n) \in(0,1)$.

Once we have a bound on $p$-accessible max-entropy for some $p<1$, we need to apply several transformations to obtain a function with a good bound on $\operatorname{neg}(n)$-accessible max-entropy.

Our second construction (which achieves better parameters), starts with a bound on a different average-case form of accessible entropy, which is stronger than bounding the accessible Shannon entropy. The benefit of this notion it that it can be converted more efficiently to $\operatorname{neg}(n)$-accessible max-entropy, by simply taking repetitions.

To motivate the definition, recall that a bound on accessible Shannon entropy means that the sample entropy $\mathrm{H}_{A(X ; R) \mid X}\left(x^{\prime} \mid x\right)$ is small on average over $x \stackrel{\mathrm{R}}{\leftarrow} X$ and $x^{\prime} \stackrel{\mathrm{R}}{\leftarrow}$ $A(x ; R)$. This sample entropy may depend on both the input $x$ and the $x^{\prime}$ output by the adversary (which in turn may depend on its coin tosses). A stronger requirement is to say that we have upper bounds $k(x)$ on the sample entropy that depend only on $x$. The following definition captures this idea, thinking of $k(x)=\log |L(x)|$. (We work with sets rather than sample entropy to avoid paying a $\log (1 / \varepsilon) \operatorname{loss}$.)

Definition 7. Let $n$ be a security parameter and $F:\{0,1\}^{n} \rightarrow\{0,1\}^{m}$ a function. We say that $F^{-1}$ has accessible average max-entropy at most $k$ if for every PPT $F$ collision-finder A, there exists a family of sets $\{L(x)\}_{x \in \operatorname{Supp}(X)}$ and a negligible 
function $\varepsilon=\varepsilon(n)$ such that $x \in L(x)$ for all $x \in \operatorname{Supp}(X), \mathrm{E}[\log |L(X)|] \leq k$ and

$$
\operatorname{Pr}[A(X ; R) \in L(X)] \geq 1-\varepsilon(n),
$$

for all sufficiently large $n$, where random variable $X$ is uniformly distributed on $\{0,1\}^{n}$ and $R$ is uniformly random coin tosses for $A$.

We observe that bounding accessible average max-entropy is indeed stronger than bounding accessible Shannon entropy:

Proposition 3. If $F^{-1}$ has accessible average max-entropy at most $k$, then for every constant $c, F^{-1}$ has accessible Shannon entropy at most $k+1 / n^{c}$.

\section{Inaccessible Entropy from One-Way Functions}

In Section 3.1 we show that any one-way function can be very slightly altered into a function with inaccessible entropy. In Section 3.2 we show that an additional hashing step implies a stronger form of inaccessible entropy (which we can then use for a more efficient construction of UOWHF). Still, we find the more direct construction of Section 3.1 and its analysis to be striking in its simplicity.

\subsection{A Direct Construction}

Theorem 1 (Inaccessible Shannon entropy from one-way functions). Let $f:\{0,1\}^{n} \mapsto\{0,1\}^{n}$ be a one-way function and define $F$ over $\{0,1\}^{n} \times[n]$ as $F(x, i)=f(x)_{1, \ldots, i}$. Then $F^{-1}$ has accessible Shannon entropy at most $\mathrm{H}(Z \mid F(Z))-1 /\left(2^{9} \cdot n^{4} \cdot \log ^{2} n\right)$, where $Z=(X, I)$ is uniformly distributed over $\left.\{0,1\}^{n} \times[n]\right]^{4}$

Proof. Suppose on the contrary that there exists a PPT $F$-collision-finder $A$ such that

$$
\mathrm{H}(Z \mid F(Z))-\mathrm{H}(A(Z ; R) \mid Z)<\varepsilon=1 /\left(2^{9} \cdot n^{4} \cdot \log ^{2} n\right)
$$

for infinitely many $n$ 's, and $R$ is uniformly distributed over the random coins of $A$. Since $I$ is determined by $F(Z)$, and since $Z$ also determines the second part of $A$ 's output (since $A$ is an $F$-collision-finder), it follows that

$$
\mathrm{H}(X \mid F(Z))-\mathrm{H}\left(A^{\prime}(Z ; R) \mid Z\right)<\varepsilon
$$

where $A^{\prime}$ is the algorithm that on input $(z ; r)$ outputs the first component of $A(z ; r)$ 's output. In the following use $A^{\prime}$ to construct an efficient algorithm that inverts $f$ with constant probability. We do so in two steps: 1. Constructing such an inverter under the assumption that we have access to an (inefficient) oracle $S a m_{\text {Ideal }}$ defined shortly, and 2. Showing how to efficiently approximate $S a m_{\text {Ideal }}$ using $A^{\prime}$.

\footnotetext{
${ }^{4}$ We believe that the actual gap between the real and accessible entropy of $F^{-1}$ is $\Omega\left(1 / n^{2}\right)$, or possibly even $\Omega(1 / n)$, and not $\Omega\left(1 / n^{4}\right)$ as stated. Since even the optimistic $\Omega(1 / n)$ bound does not yield as efficient overall construction as the one resulting from Section 3.2 , we defer a tighter analysis to the final version of the paper.
} 
Algorithm 1 ( Sam $\left._{\text {Ideal }}\right)$

Input: $x \in\{0,1\}^{n}, i \in[n]$ and $b \in\{0,1\}$.

Return a random $x^{\prime} \in F^{-1}(F(x, i-1))_{1}$ such that $f\left(x^{\prime}\right)_{i}=b$ (return an arbitrarily value if no such $x^{\prime}$ exists), where $F^{-1}(F(x, j))_{1}=$ $\left\{x^{\prime} \in\{0,1\}^{n}: F\left(x^{\prime}, j\right)=F(x, j)\right\}$.

That is, $\operatorname{Sam}_{\text {Ideal }}$ outputs uniformly at random $x^{\prime}$ such that $f\left(x^{\prime}\right)_{1, \ldots, i}=$ $\left(f(x)_{1, \ldots, i-1}, b\right)$. We define an algorithm Inv with access to an oracle Sam. When $S a m=S a m_{\text {Ideal }}$, it will be easy to argue that Inv inverts $f$ with probability one.

Algorithm 2 (Inv $\left.v^{\text {Sam }}\right)$

Input: $y \in\{0,1\}^{n}$.

Oracle: $\mathrm{Sam}$.

For $i=1$ to $n$ do:

let $x^{i}=\operatorname{Sam}\left(x^{i-1}, i, y_{i}\right)$ (where $x^{0}$ is chosen arbitrarily)

Output $x^{n}$.

It is immediate that $\operatorname{Inv} v^{\operatorname{Sam}_{\text {Ideal }}}$ inverts $f$ with probability one. We now turn to showing that $A^{\prime}$ can be used to efficiently approximate $S a m_{\text {Ideal }}$. The resulting algorithm $\operatorname{Sam}_{\delta}$ will be sufficiently similar to $\operatorname{Sam}_{\text {Ideal }}$ and as a result $\operatorname{Inv} \mathrm{Sam}_{\delta}$ will still invert $f$ with high probability. A property of Inv that will come handy is that, on a uniform value $y=f(x)$, the first coordinate of each individual query that the inverter

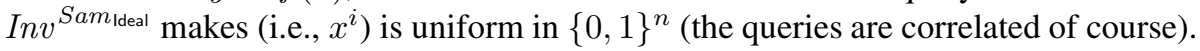

Recall that the output of $A^{\prime}$ has high Shannon entropy - almost as high as the uniform distribution over its set of prescribed outputs. Claim 3.1 (which is rather standard), shows that this also implies that the distribution of $A^{\prime}$ 's output is statistically close to this uniform distribution.

Definition 8. For $\delta \in[0,1]$ let $\mathcal{A}_{\delta}$ be the family of efficient $F$-collision-finders with the following guarantee: for every $A^{\prime \prime} \in \mathcal{A}_{\delta}$ there exist infinitely many $n$ 's such that $\left\|\left(Z, A^{\prime \prime}(Z ; R)\right)-\left(Z, \mathrm{~F}^{-1}(F(Z))_{1}\right)\right\| \leq \delta$, where $R$ is uniformly distributed over the random-coins of $A^{\prime \prime}$ and $\mathrm{F}^{-1}(F(x, i))_{1}$ is uniformly distributed over $F^{-1}(F(x, i))_{1}$.

Showing that the output of $A^{\prime}$ is statistically close to uniform can therefore be formalized by showing the following claim:

Claim. $A^{\prime} \in \mathcal{A}_{\sqrt{\varepsilon}}$.

Proof.

$$
\begin{aligned}
\left\|\left(Z, \mathrm{~F}^{-1}(F(Z))_{1}\right)-\left(Z, A^{\prime}(Z ; R)\right)\right\| & \left.=\underset{z \leftarrow Z}{\mathrm{E}}\left[\| \mathrm{F}^{-1}(F(z))_{1}-A^{\prime}(z ; R)\right) \|\right] \\
& \leq \underset{z \leftarrow Z}{\mathrm{E}}\left[\sqrt{\mathrm{H}\left(\mathrm{F}^{-1}(F(z))_{1}\right)-\mathrm{H}\left(A^{\prime}(z ; R)\right)}\right] \\
& \leq \sqrt{\underset{z \leftarrow Z}{\mathrm{E}\left[\mathrm{H}\left(\mathrm{F}^{-1}(F(z))_{1}\right)-\mathrm{H}\left(A^{\prime}(z ; R)\right)\right]}} \\
& =\sqrt{\mathrm{H}(X \mid F(Z))-\mathrm{H}\left(A^{\prime}(Z ; R)\right)} \\
& \leq \sqrt{\varepsilon},
\end{aligned}
$$


where the first inequality uses the fact that if $W$ is a random variable whose support is contained in a set $S$ and $U$ is the uniform distribution on $S$, then $\|U-W\| \leq$ $\sqrt{\mathrm{H}(U)-\mathrm{H}(W)}$. (See [2, Lemma 11.6.1].)

As we just shown that $A^{\prime} \in \mathcal{A}_{\sqrt{\varepsilon}}$ it is enough to show how to use an algorithm $A^{\prime \prime} \in \mathcal{A}_{\delta}$ to approximate $\operatorname{Sam}_{\text {Ideal }}$ (with error which depends on $\delta$ ). In order to keep notation simple, we abuse notation and denote by $\mathcal{A}_{\delta}$ some $A^{\prime \prime} \in \mathcal{A}_{\delta}$. Fix $\delta \in[0,1]$ and consider the following efficient approximation of $\operatorname{Sam}_{\text {Ideal }}$ :

\section{Algorithm $3\left(\mathrm{Sam}_{\delta}\right)$ \\ Input: $x \in\{0,1\}^{n}, i \in[n]$ and $b \in\{0,1\}$. \\ Oracle: $\mathcal{A}_{\delta}$.}

Repeat $16 n \cdot \log n$ times:

1. Let $x^{\prime}=\mathcal{A}_{\delta}(x, i)$

2. If $f\left(x^{\prime}\right)_{i}=b$, return $x^{\prime}$.

Abort.

Let $I n v_{\delta}$ denote $I n v^{S a m_{\delta}}$ and $I n v_{\text {Ideal }}$ denote $I n v^{\operatorname{Sam}_{\text {Ideal }}}$. We will show that the output of $I n v_{\delta}$ (on a random value $f(x)$ ) is statistically close to that of $I n v_{\text {Ideal }}$. As $I n v_{\text {Ideal }}$ inverts $f$ with probability one, we will conclude that $\operatorname{In} v_{\delta}$ inverts $f$ with high probability as well. To analyze the statistical distance between the outputs of the two inverters, we consider hybrid inverters that use the ideal $S a m_{\text {Ideal }}$ in the first queries and use $S a m_{\delta}$ in the rest of the queries: For $i \in[n+1]$ let $\operatorname{Inv}_{\delta}^{i}$ be the variant of $\operatorname{Inv}$ that uses $S a m_{\text {Ideal }}$ in the first $i-1$ queries and $\operatorname{Sam}_{\delta}$ for the rest of the queries. The next claim will allow us to easily bound the difference between the output distribution of any two neighboring hybrid inverters:

Claim. Let $i \in[n]$ and let $\delta_{i}=\left\|\left(X, \mathcal{A}_{\delta}(X, i ; R)\right)-\left(X, \mathrm{~F}^{-1}(F(X, i))_{1}\right)\right\|$, then $\left\|\left(X, \operatorname{Sam}_{\text {Ideal }}\left(X, i, f(X)_{i}\right)\right)-\left(X, \operatorname{Sam}_{\delta}\left(X, i, f(X)_{i}\right)\right)\right\| \leq 1 / 2 n+16 \cdot n \cdot \log n \cdot \delta_{i}$.

Proof. $\mathrm{Sam}_{\delta}$ is imperfect for two reasons (which our analysis handles separately). The first reason is that $\operatorname{Sam}_{\delta}$ relies on the output of $\mathcal{A}_{\delta}$ that returns an inverse that is only close to uniform (rather than fully uniform). The error accumulated in each query to $\mathcal{A}_{\delta}$ is $\delta_{i}$ and there are only $16 \cdot n \cdot \log n$ such queries, which altogether contributes $16 \cdot n \cdot \log n \cdot \delta_{i}$ to the statistical distance bounded by the claim. The second source of error is that after $16 \cdot n \cdot \log n$ unsuccessful repetitions, $\operatorname{Sam}_{\delta}$ aborts without retrieving a correct inverse $x^{\prime}$. As we now argue, such failure will only happen with small probability (contributing $\frac{1}{2 n}$ to the bound in the claim).

To separate our analysis of the two sources of error, we start by considering the case that $\delta_{i}=0$. Note that in this case $\mathcal{A}_{\delta}(x, i ; R)=\mathcal{A}_{0}(x, i ; R)$ is identical to $\mathrm{F}^{-1}(F(x, i))_{1}$. For $x \in\{0,1\}^{n}, i \in[m]$ and $b \in\{0,1\}$, let $\alpha(x, i, b):=\operatorname{Pr}_{y \stackrel{\mathrm{R}}{\leftarrow} f(X)}\left[y_{i}=b \mid y_{1, \ldots, i-1}=f(x)_{1, \ldots, i-1}\right]$. Note that for every $i$, $\left.\operatorname{Pr}\left[\alpha\left(X, i, f(X)_{i}\right)\right)<\beta\right]<\beta$ for every $\beta>0$. We also note that $\operatorname{Sam}_{\delta}\left(x, i, f(x)_{i}\right)$ aborts with probability at most $\left(1-\frac{1}{4 n}\right)^{16 n \cdot \log n}<\frac{1}{4 n}$ in the case that $\alpha\left(x, i, f(x)_{i}\right) \geq$ 
$\frac{1}{4 n}$, and that in case it does not abort, (since we assume that $\delta_{i}=0$ ) it returns the same distribution as $\operatorname{Sam}_{\text {Ideal }}(x, i, b)$ does. Hence, for the case that $\delta_{i}=0$ we have that

$$
\begin{aligned}
& \left\|\left(X, \operatorname{Sam}_{\text {Ideal }}\left(X, i, f(X)_{i}\right)\right)-\left(X, \operatorname{Sam}_{\delta}\left(X, i, f(X)_{i}\right)\right)\right\| \\
& \leq \operatorname{Pr}\left[\alpha\left(X, i, f(X)_{i}\right)<\frac{1}{4 n}\right]+\operatorname{Pr}\left[\operatorname{Sam}_{\delta}\left(X, i, f(X)_{i}\right) \text { aborts } \mid \alpha\left(X, i, f(X)_{i}\right) \geq \frac{1}{4 n}\right] \\
& <\frac{1}{4 n}+\frac{1}{4 n} \\
& \leq \frac{1}{2 n} .
\end{aligned}
$$

We now want to analyze the general case where $\delta_{i}$ may be larger than zero. The statistical distance between the output distribution of $\operatorname{Sam}_{\delta}\left(X, i, f(X)_{i}\right)$ in the case $\delta_{i}=0$ and in the general case is at most the maximal number of calls to $\mathcal{A}_{\delta}$ made by $\operatorname{Sam}_{\delta}$ times $\left\|\left(X, \mathcal{A}_{\delta}(X, i)\right)-\left(X, \mathcal{A}_{0}(X, i)\right)\right\|$, we therefore have that

$$
\begin{aligned}
& \left\|\left(X, \operatorname{Sam}_{\text {Ideal }}\left(X, i, f(X)_{i}\right)-\left(X, \operatorname{Sam}_{\delta}\left(X, i, f(X)_{i}\right)\right)\right)\right\| \\
& \leq \frac{1}{2 n}+16 n \cdot \log n \cdot \|\left(X, \mathcal{A}_{\delta}(X, i)-\left(X, \mathcal{A}_{0}(X, i) \|\right.\right. \\
& =\frac{1}{2 n}+16 n \cdot \log n \cdot\left\|\left(X, \mathcal{A}_{\delta}(X, i)-\left(X, \mathrm{~F}^{-1}(F(X, i))_{1}\right)\right)\right\| \\
& =\frac{1}{2 n}+16 \cdot n \cdot \log n \cdot \delta_{i} .
\end{aligned}
$$

Now note that the $i$ 'th query of $\operatorname{Inv}_{\delta}^{i}(f(X))$ (a query to $S a m_{\delta}$ ) and the $i$ 'th query of $\operatorname{Inv} v_{\delta}^{i+1}(f(X))$ (a query to $\operatorname{Sam}_{\text {Ideal }}$ ) are both distributed as $\left(X, i, f(X)_{i}\right)$. Therefore Claim 3.1 yields that for every $i \in[n]$,

$$
\left\|\operatorname{Inv} v_{\delta}^{i+1}(f(X))-\operatorname{Inv} v_{\delta}^{i}\right\| \leq \frac{1}{2 n}+16 \cdot n \cdot \log n \cdot \delta_{i} .
$$

Hence,

$$
\begin{aligned}
& \operatorname{Pr}\left[\operatorname{Inv}_{\delta}(f(X)) \in f^{-1}(f(X))\right] \\
& \geq 1-\sum_{i=1}^{n}\left\|\operatorname{Inv}_{\delta}^{i+1}(f(X))-\operatorname{Inv}_{\delta}^{i}(f(X))\right\| \\
& \geq 1-\sum_{i=1}^{n} \frac{1}{2 n}+16 \cdot n \cdot \log n \cdot \|\left(X, \operatorname{Sam}_{\text {Ideal }}\left(X, i, f(X)_{i}\right)-\left(X, \operatorname{Sam}_{\delta}\left(X, i, f(X)_{i}\right) \|\right.\right. \\
& \geq \frac{1}{2}-16 \cdot n^{2} \cdot \log n \cdot \delta .
\end{aligned}
$$

Let Inv be the instantiation of $I n v_{\delta}$ obtained when we implement $\operatorname{Sam}_{\delta}$ using $A^{\prime}$. Claim 3.1 yields that $\operatorname{Pr}\left[\operatorname{Inv}(f(X)) \in f^{-1}(f(X))\right] \geq \operatorname{Pr}\left[\operatorname{Inv}_{\sqrt{\varepsilon}}(f(X)) \in\right.$ $\left.f^{-1}(f(X))\right] \geq 1 / 2-16 \cdot n^{2} \cdot \log n \cdot \sqrt{\varepsilon}>1 / 4$. 


\subsection{A More Efficient Construction}

The following theorem shows that a simplified variant of the first step of [11] (which is also the first step of [9]) yields inaccessible entropy with much stronger guarantees than those obtained in Section 3.1. The function we construct is $F(x, g, i)=$ $\left(g(f(x))_{1, \ldots, i}, g\right)$, where $g:\{0,1\}^{n} \rightarrow\{0,1\}^{n}$ is a three-wise independent function. Since the composition of $g$ and $f$ is still a one-way function then Theorem 1 already implies that $F^{-1}$ has inaccessible entropy. The benefits of the additional hashing step are that 1 . we get more inaccessible entropy $\left(\tilde{\Theta}(1 / n)\right.$ bits rather than $\tilde{\Theta}\left(1 / n^{4}\right)$ bits), and 2 . we get a bound on accessible average max-entropy rather than accessible Shannon entropy. These allow for a simpler and more efficient transformation of $F$ into a UOWHF.

Theorem 2 (Inaccessible average max-entropy from one-way functions). Let $f:\{0,1\}^{n} \mapsto\{0,1\}^{n}$ be a one-way function and let $\mathcal{G}=\left\{g:\{0,1\}^{n} \rightarrow\{0,1\}^{n}\right\}$ be a family of constructible 5 three-wise independent hash functions. Define $F$ with domain $\operatorname{Dom}(F):=\{0,1\}^{n} \times \mathcal{G} \times[n]$ by

$$
F(x, g, i)=\left(g(f(x))_{1, \ldots, i}, g\right) .
$$

Then, for every constant $d, F^{-1}$ has accessible average max-entropy at most $\mathrm{H}(Z \mid F(Z))-(d \log n) / n$ for every $d>0$, where $Z=(X, G, I)$ is uniformly distributed over $\operatorname{Dom}(F)$.

Proof. Let $c$ be a sufficiently large constant (whose value we determine later, depending on the constant $d$ in the theorem statement) and define for every $y \in\{0,1\}^{n}$ and $i \in[n]$ :

$$
L(y, i)=\left\{y^{\prime} \in\{0,1\}^{n}: \mathrm{H}_{f(X)}\left(y^{\prime}\right) \geq(i+c \cdot \log n) \vee y^{\prime}=y\right\} .
$$

(Recall that the sample entropy is defined as $\mathrm{H}_{f(X)}(y)=\log (1 / \operatorname{Pr}[f(X)=y])=n-$ $\log \left|f^{-1}(y)\right|$, so the "heavy" images, where $f^{-1}(y)$ is large, have low sample entropy.) Namely, $L(y, i)$ consists, in addition to $y$ itself, of " $i$-light" images with respect to $f$.

We later show that the sets $L^{\prime}(x, g, i)=f^{-1}(L(f(x), i)) \times\{(g, i)\}$ satisfy the properties required to show that the accessible max-entropy of $F^{-1}$ is as stated in the theorem 6 Towards this goal, we first show that the only accessible inputs of $F$ come from preimages of $L(y, i)$.

Claim. For every PPT $F$-collision-finder $A$ and every constant $c>0$, it holds that

$$
\operatorname{Pr}\left[A_{1}(X, G, I ; R) \notin f^{-1}(L(f(X), I))\right] \leq \operatorname{neg}(n),
$$

where $(X, G, I)$ is uniformly distributed over $\operatorname{Dom}(F), R$ is uniformly distributed over the random coins of $A$, and $A_{1}$ denotes the first component of $A$ 's output.

${ }^{5} \mathcal{G}$ is constructible if given the description of a function $g \in \mathcal{G}$ and $x \in\{0,1\}^{n}, g(x)$ can be computed in time poly $(n)$, and there is a probabilistic polynomial-time algorithm that given $x \in\{0,1\}^{n}$, and $y \in\{0,1\}^{n}$, outputs a random $g \stackrel{\mathrm{R}}{\leftarrow} \mathcal{G}$ such that $g(x)=y$.

${ }^{6}$ We are working with the set $L$, and not with $L^{\prime}$, as it significantly simplifies notations. Note that the sets $L^{\prime}$ are independent of the adversary, even though the definition of accessible average max-entropy allows the sets to depend on the adversary. Further, note that the sets $L$ are independent of $\mathcal{G}$. 
Note that the above immediately yields that $\operatorname{Pr}\left[A(X, G, I ; R) \notin L^{\prime}(X, G, I)\right] \leq$ $\operatorname{neg}(n)$, since the other two output components of $A$ are required to equal $(g, i)$, due to the fact that $F(x, g, i)$ determines $(g, i)$.

Proof. Suppose on the contrary that there exist an efficient $F$-collision-finder $A$, $c>0$ and a non-negligible function $\varepsilon=\varepsilon(n)$ such that $\operatorname{Pr}\left[A_{1}(X, G, I ; R) \notin\right.$ $\left.f^{-1}(L(f(X), I))\right] \geq \varepsilon$. Fix a triple $(x, i, r)$ and let

$$
\varepsilon_{x, i, r}=\operatorname{Pr}\left[A_{1}(x, G ; r) \notin f^{-1}(L(f(x), i))\right] .
$$

Define $A^{\prime}(g)=A_{1}(x, g ; r)$. We will show how to use any such $A^{\prime}$ to invert $f$ with probability at least $\varepsilon_{x, i, r} / n^{c}$. By picking $(x, i, r)$ at random, we will invert $f$ with probability at least $\mathrm{E}_{x, i, r}\left[\varepsilon_{x, i, r} / n^{c}\right]=\varepsilon / n^{c}$, which contradicts the one-wayness of $f$. Our inverter works as follows, on input $y \in\{0,1\}^{n}$.

$\operatorname{Inv}(y)$ : choose $g$ uniformly at random from $\mathcal{G}$ subject to the constraint $g(y)_{1 \ldots i}=$ $\left.g(f(x))_{1 \cdots i}\right]^{7}$ and output $A^{\prime}(g)$.

To analyze the success probability Inv, we first rewrite the success probability of $A^{\prime}$ as follows:

$$
\begin{aligned}
\varepsilon_{x, i, r} \leq & \operatorname{Pr}\left[A^{\prime}(G) \notin f^{-1}(L(f(x), i))\right. \\
= & \sum_{y \notin L(f(x), i)} \operatorname{Pr}\left[A^{\prime}(G) \in f^{-1}(y)\right] \\
= & \sum_{y \notin L(f(x), i)} \operatorname{Pr}\left[G(y)_{1 \cdots i}=G(f(x))_{1 \cdots i}\right] \\
& \quad \cdot \operatorname{Pr}\left[A^{\prime}(G) \in f^{-1}(y) \mid G(y)_{1 \cdots i}=G(f(x))_{1 \cdots i}\right] \\
= & 2^{-i} \cdot \sum_{y \notin L(f(x), i)} \operatorname{Pr}\left[A^{\prime}(G) \in f^{-1}(y) \mid G(y)_{1 \cdots i}=G(f(x))_{1 \cdots i}\right] .
\end{aligned}
$$

Above the second equality follows because $A$ is an $F$-collision finder (so it is always the case that $x^{\prime}=A^{\prime}(g)=A(x, g, i)_{1}$ has the property that $\left.g\left(f\left(x^{\prime}\right)\right)_{1 \cdots i}=g(f(x))_{1 \cdots i}\right)$, and the third inequality follows by the two-wise independence of $\mathcal{G}$ ( $y \notin L(f(x), i)$ implies that $y \neq f(x)$ ). Now, we can bound the success probability of Inv in finding a preimage of $Y=f(X)$ by:

$$
\begin{aligned}
& \operatorname{Pr}\left[\operatorname{Inv}(Y) \in f^{-1}(Y)\right] \\
& =\sum_{y} \operatorname{Pr}[Y=y] \cdot \operatorname{Pr}\left[A^{\prime}(G) \in f^{-1}(y) \mid G(y)_{1 \cdots i}=f(x)_{1 \cdots i}\right] \\
& \geq \sum_{y \notin L(f(x), i)} \operatorname{Pr}[Y=y] \cdot \operatorname{Pr}\left[A^{\prime}(G) \in f^{-1}(y) \mid G(y)_{1 \cdots i}=f(x)_{1 \cdots i}\right] \\
& \geq \frac{1}{2^{i+c \log n}} \cdot \sum_{y \notin L(f(x), i)} \operatorname{Pr}\left[A^{\prime}(G) \in f^{-1}(y) \mid G(y)_{1 \cdots i}=f(x)_{1 \cdots i}\right] \\
& \geq \varepsilon_{x, i, r} / n^{c},
\end{aligned}
$$

\footnotetext{
${ }^{7}$ This can be done by first choosing $z \stackrel{R}{\leftarrow}\{0,1\}^{n-i}$ and then using the constructibility of $\mathcal{G}$ to
} generate a random $g$ such that $g(y)=\left(g(f(x))_{1 \cdots i}, z\right)$. 
where the penultimate inequality holds because every $y \notin L(f(x), i)$ satisfies $\mathrm{H}_{f(X)}(y)<(i+c \cdot \log n)$.

We have seen that sets $f^{-1}(L(y, i))$ capture the accessible inputs of $F$; now it remains to show that the expected logarithm of their size is sufficiently smaller than the real entropy $\mathrm{H}(Z \mid F(Z))=\mathrm{E}\left[\log \left|F^{-1}(F(Z))\right|\right]$ (again, this property immediately propagates to $L^{\prime}$ ).

Claim. For every constant $c>8$, it holds that

$$
\mathrm{E}\left[\log \left|f^{-1}(L(f(X), I))\right|\right] \leq \mathrm{E}\left[\log \left|F^{-1}(F(Z))\right|\right]-\Omega\left(\frac{c \log n}{n}\right),
$$

where $Z=(X, G, I)$ is uniformly distributed in $\operatorname{Dom}(F)$.

Proof. We assume for simplicity that $n$ is a power of 2 (otherwise, we "pad" $f$ ) and that $c$ is a power of 2 , and let $c^{\prime}=c / 2$. For ease of notation, we will work in entropy units of $c^{\prime} \log n$. Namely, for $i \in\left\{0, \cdots, m=n /\left(c^{\prime} \log n\right)\right\}$ and $y \in\{0,1\}^{n}$, let $y_{\{1\}, \ldots,\{i\}}$ be the first $i \cdot c^{\prime} \log n$ bits of $y$, define

$$
\mathrm{H}_{f}(y):=\frac{\mathrm{H}_{f(X)}(y)}{c^{\prime} \log n} .
$$

and let

$$
q_{i}=\operatorname{Pr}\left[\mathrm{H}_{f}(f(X)) \in[i, i+1)\right] .
$$

Recall that $(X, G, I)$ is uniformly distributed in $\operatorname{Dom}(F)$. We define additional random variables that categorize the "non trivial collisions" induced by $F$ into two separate categories:

$$
\begin{aligned}
& \text { Light }:=\mid\left\{x^{\prime} \in\{0,1\}^{n}: f\left(x^{\prime}\right) \neq f(X) \wedge G\left(f\left(x^{\prime}\right)\right)_{\{1\}, \ldots,\{I\}}=G(f(X))_{\{1\}, \cdots,\{I\}}\right. \\
& \left.\quad \wedge \mathrm{H}_{f}\left(f\left(x^{\prime}\right)\right) \geq I+2\right\} \mid .
\end{aligned}
$$

Namely, Light consists of the preimages that collide with $f(X)$, different from $f(X)$, and "light" — have few preimages. Similarly, let

$$
\begin{aligned}
& \text { Heavy }:=\mid\left\{x^{\prime} \in\{0,1\}^{n}: f\left(x^{\prime}\right) \neq f(X) \wedge G\left(f\left(x^{\prime}\right)\right)_{\{1\}, \ldots,\{I\}}=G(f(X))_{\{1\}, \cdots,\{I\}}\right. \\
& \left.\quad \wedge \mathrm{H}_{f}\left(f\left(x^{\prime}\right)\right)<I+2\right\} \mid .
\end{aligned}
$$

Namely, Heavy consists of the preimages that collide with $f(X)$, different from $f(X)$, and "heavy" — have many preimages. Note that

$$
\left|F^{-1}(F(Z))\right|=\text { Light }+ \text { Heavy }+\left|f^{-1}(f(X))\right|
$$

(recall that the all elements $F^{-1}(F(x, g, i))$ are of the form $\left.\left(x^{\prime}, g, i\right)\right)$ and

$$
\left|f^{-1}(L(f(X), I))\right| \leq \text { Light }+\left|f^{-1}(f(X))\right| .
$$


Thus, we have

$$
\begin{aligned}
& \mathrm{E}\left[\log \left|F^{-1}(F(Z))\right|\right]-\mathrm{E}\left[\log \left|f^{-1}(L(f(X), I))\right|\right] \\
& \geq \mathrm{E}\left[\log \frac{\text { Light }+ \text { Heavy }+\left|f^{-1}(f(X))\right|}{\text { Light }+\left|f^{-1}(f(X))\right|}\right]
\end{aligned}
$$

We manipulate this as follows:

$$
\begin{aligned}
& \mathrm{E}\left[\log \frac{\text { Light }+ \text { Heavy }+\left|f^{-1}(f(X))\right|}{\left|f^{-1}(f(X))\right|+\text { Light }}\right] \\
& \geq \mathrm{E}\left[\log \left(1+\frac{\text { Heavy }}{\left|f^{-1}(f(X))\right|+\text { Light }+ \text { Heavy }}\right)\right] \\
& \geq \mathrm{E}\left[\frac{\text { Heavy }}{\left|f^{-1}(f(X))\right|+\text { Light }+ \text { Heavy }}\right],
\end{aligned}
$$

where the last inequality uses the fact that $\log (1+\alpha) \geq \alpha$ for $\alpha \leq 1$. The proof of Claim 3.2 easily follows from the next claim, which yields that with constant probability, Heavy is a significant term in $\left(\left|f^{-1}(f(X))\right|+\right.$ Light + Heavy $)$

Claim. Let $\alpha \geq 1, i \in\{0, \ldots, m-1\}$ and $x \in\{0,1\}^{n}$. Condition on $I=i$ and $X=x$, and define the following events (over the random variable $G$ ):

$$
\begin{aligned}
& E_{i}^{1}:(\text { Light }+ \text { Heavy }) \leq 3 \cdot 2^{n-i \cdot\left(c^{\prime} \log n\right)} \\
& E_{i}^{2}: \text { Heavy } \geq\left(q_{i+1}-\alpha \cdot \sqrt{1 / n^{c^{\prime}}}\right) \cdot 2^{n-i \cdot c^{\prime} \log n-1}
\end{aligned}
$$

Then $\operatorname{Pr}\left[E_{i}^{1}\right] \geq 2 / 3$, and $\operatorname{Pr}\left[E_{i}^{2}\right] \geq 1-4 / \alpha^{2}$.

Proof. For $E_{i}^{1}$, we note that E[Light + Heavy $] \leq 2^{n-i \cdot\left(c^{\prime} \log n\right)}$ by two-universality of $\mathcal{G}$, and apply Markov's Inequality.

For $E_{i}^{2}$, let

$$
S:=\left\{x^{\prime} \in\{0,1\}^{n}: f\left(x^{\prime}\right) \neq f(x) \wedge \mathrm{H}_{f}\left(f\left(x^{\prime}\right)\right) \in[i+1, i+2)\right\} .
$$

Note that $|S| \geq\left(q_{i+1}-\operatorname{neg}(n)\right) \cdot 2^{n}$, where we subtract neg $(n)$ for not taking into account the preimages of $f(x)$. For $g \in \mathcal{G}$, let

$$
\begin{aligned}
S_{g}:=\left\{x^{\prime} \in\{0,1\}^{n}:\right. & f\left(x^{\prime}\right) \neq f(x) \wedge \mathrm{H}_{f}\left(f\left(x^{\prime}\right)\right) \in[i+1, i+2) \\
& \left.\wedge g\left(f\left(x^{\prime}\right)\right)_{\{1\}, \ldots,\{i\}}=g(f(x))_{\{1\}, \cdots,\{i\}}\right\},
\end{aligned}
$$

note that (conditioned on $I=i$ and $X=x$ ) Heavy $\geq\left|S_{G}\right|$. We write $\left|S_{g}\right|=\sum_{y \in f(S)} 1_{g, y} \cdot\left|f^{-1}(y)\right|$, where $1_{g, y}$ is the indicator for $g(y)_{\{1\}, \ldots,\{i\}}=$

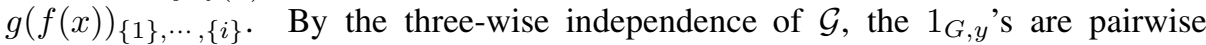
independent Bernoulli random variables, each with expectation $2^{-i \cdot c^{\prime} \log n}$. Thus, $\mathrm{E}\left[\left|S_{G}\right|\right] \geq\left(q_{i+1}-\operatorname{neg}(n)\right) \cdot 2^{n-i \cdot c^{\prime} \log n}$. Assuming that $q_{i+1}>\alpha \cdot \sqrt{1 / n^{c^{\prime}}} \geq \sqrt{1 / n^{c^{\prime}}}$ (as otherwise the claim about $E_{i}^{2}$ holds trivially), it follows that

$$
\mathrm{E}\left[\left|S_{G}\right|\right]>q_{i+1} \cdot 2^{n-i \cdot c^{\prime} \log n-1}
$$


By the pairwise independence of $1_{G, y}$ 's, we also have

$$
\begin{aligned}
& \operatorname{Var}\left[\left|S_{G}\right|\right]=\sum_{y \in f(S)} \operatorname{Var}\left[1_{G, y} \cdot\left|f^{-1}(y)\right|\right] \\
& \leq 2^{-i \cdot c^{\prime} \log n} \cdot \sum_{y \in f(S)}\left|f^{-1}(y)\right|^{2} \\
& \leq 2^{-i \cdot c^{\prime} \log n} \cdot|S| \cdot \max _{y \in f(S)}\left|f^{-1}(y)\right| \leq 2^{-i \cdot c^{\prime} \log n} \cdot 2^{n} \cdot 2^{n-(i+1) \cdot c^{\prime} \log n} \\
& =\left(\frac{2}{\sqrt{n^{c^{\prime}}}} \cdot 2^{n-i \cdot c^{\prime} \log n-1}\right)^{2},
\end{aligned}
$$

and thus by Chebyshev inequality

$$
\begin{aligned}
\operatorname{Pr}\left[E_{i}^{2}\right] & \geq \operatorname{Pr}\left[\left|S_{G}\right| \geq\left(q_{i+1}-\alpha \cdot \sqrt{1 / n^{c^{\prime}}}\right) \cdot 2^{n-i \cdot c^{\prime} \log n-1}\right] \\
& \geq 1-\operatorname{Pr}\left[|| S_{G}\left|-\mathrm{E}\left[\left|S_{G}\right|\right]\right| \geq \frac{\alpha}{2} \cdot \sqrt{\operatorname{Var}\left[\left|S_{G}\right|\right]}\right] \geq 1-\frac{4}{\alpha^{2}} .
\end{aligned}
$$

Noting that $\mathrm{H}_{f}(f(X)) \geq i$ means $\left|f^{-1}(f(X))\right| \leq 2^{n-i \cdot\left(c^{\prime} \log n\right)}$, and applying Claim 3.2 with $\alpha=4$, we have

$$
\begin{aligned}
\mathrm{E} & {\left[\log \left|F^{-1}(F(Z))\right|\right]-\mathrm{E}\left[\log \left|f^{-1}(L(Y, I))\right|\right] } \\
\geq & \mathrm{E}\left[\frac{\text { Heavy }}{\left|f^{-1}(f(X))\right|+\text { Light }+ \text { Heavy }}\right] \\
\geq & \frac{1}{m} \cdot \sum_{i=0}^{m-1} \operatorname{Pr}\left[\mathrm{H}_{f}(f(X)) \geq i\right] \cdot \operatorname{Pr}\left[E_{i}^{1} \wedge E_{i}^{2} \mid \mathrm{H}_{f}(f(X)) \geq i\right] \\
& \cdot \frac{\left(q_{i+1}-\frac{4}{n^{c^{\prime} / 2}}\right) \cdot 2^{n-i \cdot c^{\prime} \log n-1}}{2^{n+2-i \cdot\left(c^{\prime} \log n\right)}} \\
\geq & \frac{1}{m} \cdot \sum_{i=0}^{m-1}\left(q_{i+1}+\cdots+q_{m}\right) \cdot\left(1-\frac{1}{3}-\frac{1}{4}\right) \cdot\left(\frac{q_{i+1}-4 / n^{c^{\prime} / 2}}{8}\right) \\
\geq & \frac{1}{48 m} \cdot\left(\sum_{j, i \in\{0, \ldots, m\}} q_{i} \cdot q_{j}\right)-O\left(\frac{m}{n^{c^{\prime} / 2}}\right) \\
\geq & \frac{1}{48 m}-O\left(\frac{m}{n^{c^{\prime} / 2}}\right),
\end{aligned}
$$

where the first inequality is by Equation 2, and the third inequality holds since $q_{0}=0$ for every one-way function, which implies that $\sum_{1 \leq i \leq j \leq m} q_{i} \cdot q_{j}=\sum_{0 \leq i \leq j \leq m} q_{i} \cdot q_{j} \geq$ $\frac{1}{2} \cdot \sum_{j, i \in\{0, \ldots, m\}} q_{i} \cdot q_{j}$. Thus, Claim 3.2 holds with respect to any $c=2 c^{\prime} \geq 8$.

By Claims 3.2 and 3.2 and the fact that $F(x, g, i)$ determines $g$ and $i$, the sets $L^{\prime}(x, g, i)=f^{-1}(L(f(x), i)) \times\{(g, i)\}$ satisfy the properties required to show that the accessible max-entropy of $F^{-1}$ is at most $\mathrm{H}(Z \mid F(Z))-\Omega(c(\log n) / n)$. Taking $c$ to be a sufficiently large constant times $d$, completes the proof. 


\section{UOWHFs from Inaccessible Entropy}

In this section we show how to construct a UOWHF from any efficiently computable function with a noticeable gap between real Shannon entropy and either accessible average max-entropy or accesssible Shannon entropy. Recall that the more efficient construction from Section 3.2 satisfies the former, and the more direct construction from Section 3.1 satisfies the latter. Combined with these constructions, we obtain two new constructions of UOWHFs from any one-way function.

In both cases, we first transform the entropy gap into a noticeable gap between real Shannon entropy and accessible max-entropy. We begin with the construction that starts from a gap between real Shannon entropy and accessible average max-entropy because the transformation involves fewer steps (and is also more efficient).

\subsection{The More Efficient UOWHF}

Starting with any one-way function $f:\{0,1\}^{n} \rightarrow\{0,1\}^{n}$, the final UOWHF has output length $O\left(n^{7}\right)$ and key length $\tilde{O}\left(n^{7}\right)$ (or $O\left(n^{5}\right)$ for both output and key lengths for a non-uniform construction). Throughout, let $s \in \omega(\log n)$ denote any superlogarithmic function.

STEP 0 (basic construction): Let $F_{0}$ denote the function from Section 3.2 That is, $F_{0}$ is defined on domain $\{0,1\}^{n} \times \mathcal{G} \times[n]$ as $F_{0}(x, g, i)=\left(g(f(x))_{1, \ldots, i}, g\right)$, where $f:\{0,1\}^{n} \mapsto\{0,1\}^{n}$ is a one-way function and $\mathcal{G}$ is a family of constructible, 3 -wise independent hash functions over $\{0,1\}^{n}$.

- $F_{0}:\{0,1\}^{\ell_{0}^{\mathrm{IN}}} \rightarrow\{0,1\}^{\ell_{0}^{\text {OUT }}}$ where $\ell_{0}^{\mathrm{IN}}=\ell_{0}^{\mathrm{IN}}(n)=O(n)$ and $\ell_{0}^{\text {OUT }}=\ell_{0}^{\text {OUT }}(n)=$ $O(n)$.

- Let $k_{\text {REAL }}$ denote the real Shannon entropy of $F_{0}^{-1}$. Theorem 2 yields that the accessible average max-entropy of $F_{0}^{-1}$ is bounded by $k_{\mathrm{REAL}}-\Delta$ for $\Delta=$ $(\log n) / n$.

STEP 1 (gap amplification): Let $F_{1}$ be the $t$-fold direct product of $F_{0}$. That is, $F_{1}\left(x_{1}, \ldots, x_{t}\right)=\left(F_{0}\left(x_{1}\right), \ldots, F_{0}\left(x_{t}\right)\right)$ where $t \in O\left(n^{2} s / \Delta^{2}\right)$. Specifically, we require that

$$
t k_{\mathrm{REAL}}-\ell_{0}^{\mathrm{IN}} \cdot \sqrt{s t} \geq t \cdot\left(k_{\mathrm{REAL}}-\Delta / 2\right)+\ell_{0}^{\mathrm{IN}} \cdot \sqrt{s t}+3 s .
$$

This repetition increases both the real and accessible entropies of $F_{1}$ by a factor of $t$ (comparing to $F_{0}$ ). In addition, this repetition converts real Shannon entropy to real min-entropy and accessible average max-entropy to accessible max-entropy (up to additive terms that are sub-linear in $t$ ). More precisely:

- $F_{1}:\{0,1\}^{\ell_{1}^{\mathrm{IN}}} \rightarrow\{0,1\}^{\ell_{1}^{\mathrm{OUT}}}$ where $\ell_{1}^{\mathrm{IN}}(n)=t \cdot \ell_{0}^{\mathrm{IN}}=O(t n)$ and $\ell_{1}^{\text {OUT }}(n)=$ $t \cdot \ell_{0}^{\mathrm{OUT}}=O(t n)$.

- $F_{1}^{-1}$ has real min-entropy at least $t k_{\mathrm{REAL}}-\ell_{0}^{\mathrm{OUT}} \cdot \sqrt{s t}$, which by our choice of $t$ is at least $t \cdot\left(k_{\mathrm{REAL}}-\Delta / 2\right)+\ell_{0}^{\mathrm{IN}} \cdot \sqrt{s t}+3 s$.

- $F_{1}^{-1}$ has accessible max-entropy at most $t \cdot\left(k_{\mathrm{REAL}}-\Delta\right)+\ell_{0}^{\mathrm{IN}} \cdot \sqrt{s t}$. 
From the next step on, the construction is given an additional parameter $k$ (a "good" estimate of $\left.k_{\mathrm{REAL}}\right)$ such that $k \in\left[k_{\mathrm{REAL}}, k_{\mathrm{REAL}}+\Delta / 2\right]$. This means that:

- $F_{1}^{-1}$ has real min-entropy at least $t \cdot(k-\Delta)+\ell_{0}^{\text {out }} \cdot \sqrt{s t}+3 s$.

- $F_{1}^{-1}$ has accessible max-entropy at most $t \cdot(k-\Delta)+\ell_{0}^{\text {out }} \cdot \sqrt{s t}$.

That is, there is a gap of $3 s$ between real min-entropy and accessible max-entropy, and moreover, we "know" where the gap is (given $k$ ).

STEP 2 (entropy reduction): Apply entropy reduction to $F_{1}$ to obtain $F_{2}$. That is, $F_{2}(x, g)=\left(F_{1}(x), g, g(x)\right)$, where $g:\{0,1\}^{\ell_{1}^{\mathrm{IN}}} \rightarrow\{0,1\}^{\ell}$ is selected from a family of 2-universal hash functions, where $\ell=\ell(n, k)=t \cdot(k-\Delta)+\ell_{0}^{\mathrm{IN}} \cdot \sqrt{s t}+s=$ $O(t n)$. This additional hashing reduces the real min-entropy and accessible maxentropy by $\ell$ (up to an additive term of $s$ ). More precisely,

- $F_{2}:\{0,1\}^{\ell_{2}^{\mathrm{IN}}} \rightarrow\{0,1\}^{\ell_{2}^{\text {ouT }}}$ where $\ell_{2}^{\mathrm{IN}}(n, k)=O(t n)$ and $\ell_{2}^{\text {OUT }}(n, k)=O(t n)$.

- $F_{2}^{-1}$ has real min-entropy at least $s$.

- $F_{2}^{-1}$ has accessible max-entropy at most 0 . Hence, $F_{2}$ is collision-resistant on random inputs (by Lemma 1).

STEP 3 (reducing the output length): First reduce the output length of $F_{2}$ by hashing the output to $\ell_{2}^{\mathrm{IN}}-\log n$ bits. That is, $F_{3}(x, g)=\left(g, g\left(F_{3}(x)\right)\right)$ where $g$ : $\{0,1\}^{\ell_{2}^{\text {OUT }}} \rightarrow\{0,1\}^{\ell_{2}^{\mathrm{IN}}-\log n}$ is selected from a family of pairwise-independent hash functions.

- $F_{3}:\{0,1\}^{\ell_{3}^{\mathrm{IN}}} \rightarrow\{0,1\}^{\ell_{3}^{\mathrm{ouT}}}$ where $\ell_{3}^{\mathrm{IN}}(n, k)=O(t n)$ and $\ell_{3}^{\mathrm{OUT}}(n, k)=\ell_{3}^{\mathrm{IN}}-$ $\log n$.

- $F_{3}$ remains collision-resistant on random inputs.

Next, transform $F_{3}$ into a family $\left\{F_{y}\right\}$ of target collision-resistant hash functions via a random shift. That is, $F_{y}(x)=F_{3}(y+x)$.

- This yields a non-uniform construction $\left\{F_{y}\right\}$ with input length and key length $\ell_{3}^{\mathrm{IN}}(n, k)=O(t n)=O\left(n \cdot n^{2} s / \Delta^{2}\right)=O\left(n^{5}\right)$, where the non-uniformity corresponds to choice of the parameter $k \in\left[k_{\mathrm{REAL}}, k_{\mathrm{REAL}}+\Delta / 2\right]$.

STEP 4 (removing non-uniformity): To remove the non-uniform advice $k$, we "try all possibilities" from 0 to $\ell_{0}^{\mathrm{IN}}(n)$ in steps of size $\Delta / 2$, similar to the approach used in [11] (see also [9, Section 3.6]) :

i. First, we construct $m=\ell_{0}^{\mathrm{IN}}(n) \cdot 2 / \Delta$ families of functions $\left\{F_{y}^{i}\right\}$ for $i=$ $1,2, \ldots, m$, where $\left\{F_{y}^{i}\right\}$ is the family of functions obtained by instantiating Steps 1 through 3 with the parameter $k$ set to the value $i \Delta / 2$. This $m$ families of functions satisfy the following properties:

- Each of $F_{y}^{1}, \ldots, F_{y}^{m}$ is length-decreasing; in particular, $F_{y}^{i}$ has input length $\ell_{3}^{\mathrm{IN}}(n, i \Delta / 2)$ and output length $\ell_{3}^{\mathrm{IN}}(n, i \Delta / 2)-\log n$. Note that $\ell_{3}^{\mathrm{IN}}(n, i \Delta / 2) \leq \ell_{3}^{\mathrm{IN}}\left(n, \ell_{0}^{\mathrm{IN}}(n)\right)$ for all $i$ because $\ell(n, k)$ increases as a function of $k$. We may then assume that all $m$ functions $F_{y}^{1}, \ldots, F_{y}^{m}$ have the same input length $\ell_{3}^{\mathrm{IN}}\left(n, \ell_{0}^{\mathrm{IN}}(n)\right)$ and the same output length $\ell_{3}^{\mathrm{IN}}\left(n, \ell_{0}^{\mathrm{IN}}(n)\right)-\log n$ by padding "extra part" of the input to the output.

- At least one of $\left\{F_{y}^{1}\right\}, \ldots,\left\{F_{y}^{m}\right\}$ is target collision-resistant; this is because $k_{\text {REAL }} \in\left[0, \ell_{0}^{\mathrm{IN}}(n)\right]$ so there exists some $i$ for which $i \Delta / 2$ lies between $k_{\text {REAL }}$ and $k_{\text {REAL }}+\Delta / 2$. 
ii. Next, for each $i=1,2, \ldots, m$, we construct a family of functions $\left\{\tilde{F}_{\tilde{y}}^{i}\right\}$ from $\left\{F_{y}^{i}\right\}$ with input length $m \cdot \ell_{3}^{\mathrm{IN}}\left(n, \ell_{0}^{\mathrm{IN}}(n)\right)$, key length $O\left(\ell_{3}^{\mathrm{IN}}\left(n, \ell_{0}^{\mathrm{IN}}(n)\right) \cdot \log n\right)$ and output length $\ell_{3}^{\mathrm{IN}}\left(n, \ell_{0}^{\mathrm{IN}}(n)\right)-\log n$, by following the construction given in [13]. Again, at least one of $\left\{\tilde{F}_{\tilde{y}_{1}}^{1}\right\}, \ldots,\left\{\tilde{F}_{\tilde{y}_{m}}^{m}\right\}$ is target collision-resistant.

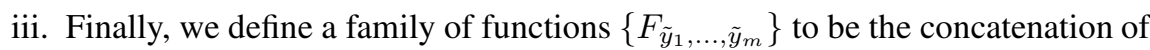
$\tilde{F}_{\tilde{y}_{1}}^{1}, \ldots, \tilde{F}_{\tilde{y}_{m}}^{m}$ on the same input. That is, $F_{\tilde{y}_{1}, \ldots, \tilde{y}_{m}}(x)=\tilde{F}_{\tilde{y}_{1}}^{1}(x) \circ \cdots \circ \tilde{F}_{\tilde{y}_{m}}^{m}(x)$.

- Note that $F$ has input length $m \cdot \ell_{3}^{\mathrm{IN}}\left(n, \ell_{0}^{\mathrm{IN}}(n)\right)$ and output length $m$. $\left(\ell_{3}^{\mathrm{IN}}\left(n, \ell_{0}^{\mathrm{IN}}(n)\right)-\log n\right)$, so $F$ is length-decreasing.

- Moreover, since at least one of $\left\{\tilde{F}_{\tilde{y}_{1}}^{1}\right\}, \ldots,\left\{\tilde{F}_{\tilde{y}_{m}}^{m}\right\}$ is target collision-

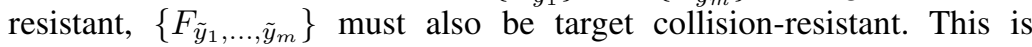
because a collision for $F_{\tilde{y}_{1}, \ldots, \tilde{y}_{m}}$ is a collision for each of $\tilde{F}_{\tilde{y}_{1}}^{1}, \ldots, \tilde{F}_{\tilde{y}_{m}}^{m}$.

This yields a uniform construction of a UOWHF with output length length $O(n / \Delta$. $\left.n \cdot n^{2} s / \Delta^{2}\right)=O\left(n^{7}\right)$. and key length $\tilde{O}\left(n / \Delta \cdot n \cdot n^{2} s / \Delta^{2} \cdot \log n\right)=\tilde{O}\left(n^{7}\right)$.

\subsection{UOWHF via a Direct Construction}

Here, the final construction has output length $\tilde{O}\left(n^{36}\right)$ (or $\tilde{O}\left(n^{26}\right)$ for a non-uniform construction).

STEP 0 (basic construction): Let $F_{0}$ denote the function from Section 3.1 . That is, $F_{0}$ is defined over $\{0,1\}^{n} \times[n]$ as $F_{0}(x, i)=\left(f(x)_{1, \ldots, i}\right)$, where $f:\{0,1\}^{n} \mapsto$ $\{0,1\}^{n}$ is a one-way function.

- $F_{0}:\{0,1\}^{\ell_{0}^{\mathrm{IN}}} \rightarrow\{0,1\}^{\ell_{0}^{\mathrm{OUT}}}$ where $\ell_{0}^{\mathrm{IN}}=\ell_{0}^{\mathrm{IN}}(n) \leq 2 n$ and $\ell_{0}^{\text {out }}=\ell_{0}^{\text {OUT }}(n) \leq$ $2 n$.

- Let $k_{\text {REAL }}$ denote the real Shannon entropy of $F_{0}^{-1}$. Theorem 1 yields that the accessible Shannon entropy of $F_{0}^{-1}$ is at most $k_{\text {REAL }}-\Delta$, where $\Delta \in \Omega\left(1 / n^{4}\right.$. $\left.\log ^{2} n\right)$

STEP 1 (gap amplification): Let $F_{1}$ be the $t$-fold direct product of $F_{0}$ for a sufficiently large $t$ to be determined later. That is, $F_{1}\left(x_{1}, \ldots, x_{t}\right)=\left(F_{0}\left(x_{1}\right), \ldots, F_{0}\left(x_{t}\right)\right)$.

This repetition increases both the real and accessible entropies of $F_{1}$ by a factor of $t$. In addition, the repetition converts real Shannon entropy to real min-entropy and real max-entropy (up to an additive $o(t)$ term). More precisely:

- $F_{1}:\{0,1\}^{\ell_{1}^{\mathrm{IN}}} \rightarrow\{0,1\}^{\text {oUT }}$ where $\ell_{1}^{\mathrm{IN}}(n)=t \cdot \ell_{0}^{\mathrm{IN}}=O(t n)$ and $\ell_{1}^{\text {OUT }}(n)=$ $t \cdot \ell_{0}^{\mathrm{OUT}}=O(t n)$.

- $F_{1}^{-1}$ has real min-entropy at least $t \cdot k_{\mathrm{REAL}}-2 n \sqrt{s t}$ and real max-entropy at most $t \cdot k_{\text {REAL }}+2 n \sqrt{s t}$.

- $F_{1}^{-1}$ has accessible Shannon entropy at most $t \cdot k_{\mathrm{REAL}}-t \Delta$.

From the next step on, the construction is given an additional parameter $k$ (a "good" estimate of $k_{\text {REAL }}$ ) such that $k \in\left[k_{\text {REAL }}, k_{\mathrm{REAL}}+\Delta^{2} / 256 n\right]$. This means that:

- $F_{1}^{-1}$ has accessible Shannon entropy at most $t k-t \Delta$. This means $F_{1}^{-1}$ has $(1-\Delta / 4 k)$-accessible max-entropy at most $t k-t \Delta / 2$. 
STEP 2 (entropy reduction): Apply entropy reduction to $F_{1}$ with $\ell=\ell(n, k)=t k-$ $t \Delta / 2+s$ to obtain $F_{2}$. That is, $F_{2}(x, g)=\left(F_{1}(x), g, g(x)\right)$, where $g:\{0,1\}^{\ell^{\mathrm{IN}}} \rightarrow$ $\{0,1\}^{\ell}$ is selected from a family of 2 -universal hash functions.

This reduces the accessible max-entropy to 0 , which allows us to deduce that $F_{2}$ is weakly collision-resistant on random inputs.

- $F_{2}:\{0,1\}_{2}^{\ell_{2}^{\text {IN }}} \rightarrow\{0,1\}_{2}^{\ell_{2}^{\text {out }}}$ where $\ell_{2}^{\text {IN }}(n, k)=O(t n+\ell(n, k))=O(t n)$ and $\ell_{2}^{\text {ouT }}(n, k)=O(t n+\ell(n, k))=O(t n)$.

$-F_{2}^{-1}$ has real min-entropy at least $t\left(k_{\mathrm{REAL}}-k+\Delta / 2\right)-2 n \sqrt{s t}-2 s$ and real max-entropy at most $t\left(k_{\mathrm{REAL}}-k+\Delta / 2\right)+2 n \sqrt{s t}$.

- $F_{2}^{-1}$ has $\left(1-\Delta / 4 k+2^{-\Omega(s)}\right)$-accessible max-entropy at most 0 . Thus, $F_{2}$ is $q$-collision-resistant on random inputsfor $q=\Delta / 4 k-2^{-\Omega(s)}$.

STEP 3 (gap amplification): $F_{3}$ is $t^{\prime}$-fold direct product of $F_{2}$, where $t^{\prime}=s / q=$ $O(k s / \Delta)=\tilde{O}\left(n^{5}\right)$. That is, $F_{3}\left(x_{1}, \ldots, x_{t^{\prime}}\right)=\left(F_{2}\left(x_{1}\right), \ldots, F_{2}\left(x_{t^{\prime}}\right)\right)$.

This allows us to amplify the weak collision-resistance property of $F_{2}$ to obtain a gap between real min-entropy and accessible max-entropy in $F_{3}$.

$-F_{3}^{-1}$ has real min-entropy at least $t^{\prime} \cdot\left(t\left(k_{\mathrm{REAL}}-k+\Delta / 2\right)-2 n \sqrt{s t}-2 s\right)$, which is at least:

$$
t^{\prime} \cdot\left(t\left(\Delta / 2-\Delta^{2} / 256 n\right)-2 n \sqrt{s t}-2 s\right) .
$$

- $F_{3}^{-1}$ has accessible max-entropy at most $t^{\prime} \cdot\left((1-q / 8)\left(t\left(k_{\mathrm{REAL}}-k+\Delta / 2\right)+\right.\right.$ $2 n \sqrt{s t})+1$, which is at most:

$$
\left.t^{\prime} \cdot(t(\Delta / 2-\Delta q / 16)+2 n \sqrt{s t})+1\right) .
$$

Now, $k \leq \ell_{0}^{\mathrm{IN}}(n) \leq 2 n$, so $q=\Delta / 4 k-2^{-\Omega(s)} \geq \Delta / 8 n-2^{-\Omega(s)}$. This means $F_{3}^{-1}$ has accessible max-entropy at most:

$$
\left.t^{\prime} \cdot\left(t\left(\Delta / 2-\Delta^{2} / 128 n+2^{-\Omega(s)}\right)+2 n \sqrt{s t}\right)+1\right) .
$$

Note that the gap is at least $t^{\prime} \cdot\left(t \cdot \Delta^{2} / 256 n-2^{-\Omega(s)}-(4 n \sqrt{s t}+2 s+1)\right)$, which is at least $3 s$ as long as:

$$
t \cdot \Delta^{2} / 256 n \geq 2^{-\Omega(s)}+4 n \sqrt{s t}+2 s+1+3 s / t^{\prime}
$$

Since $3 s / t^{\prime}=3 q \leq 3 \Delta$, we can set $t=O\left(n / \Delta+n s / \Delta^{2}+n^{4} s / \Delta^{4}\right)=\tilde{O}\left(n^{20}\right)$ so that $F_{3}^{-1}$ has a gap of $3 s$ between real min-entropy and accessible max-entropy, and moreover, we know where this gap is (given $k$ ).

STEPS 4/5/6: We follow steps 2, 3, and 4 in the previous construction, with the following modifications in the parameters:

- We apply entropy reduction first, with

$$
\left.\ell=t^{\prime} \cdot(t(\Delta / 2-\Delta q / 16)+2 n \sqrt{s t})+1\right)+s .
$$

- To remove the non-uniform advice $k$, we "try all possibilities" from 0 to $\ell_{0}^{\mathrm{IN}}(n) \leq 2 n$ in steps of size $\Delta^{2} / 256 n$.

We then obtain a non-uniform construction of UOWHFs with output length $O(n$. $\left.t \cdot t^{\prime}\right)=\tilde{O}\left(n^{26}\right)$ and a uniform construction with output length $O\left(n /\left(\Delta^{2} / n\right) \cdot n\right.$. $\left.t \cdot t^{\prime} \cdot \log n\right)=\tilde{O}\left(n^{36}\right)$. 


\section{Acknowledgements}

We are thankful to Ran Raz and Chiu-Yuen Koo for useful conversations.

\section{References}

[1] Canetti, R., Rivest, R.L., Sudan, M., Trevisan, L., Vadhan, S.P., Wee, H.: Amplifying collision resistance: A complexity-theoretic treatment. In: Menezes, A. (ed.) CRYPTO 2007. LNCS, vol. 4622, pp. 264-283. Springer, Heidelberg (2007)

[2] Cover, T.M., Thomas, J.A.: Elements of information theory, 2nd edn. Wiley-Interscience, New York (2006)

[3] Cramer, R., Shoup, V.: Design and analysis of practical public-key encryption schemes secure against adaptive chosen ciphertext attack. SIAM Journal on Computing 33(1), 167-226 (2003) (electronic)

[4] Haitner, I., Nguyen, M., Ong, S.J., Reingold, O., Vadhan, S.: Statistically hiding commitments and statistical zero-knowledge arguments from any one-way function. SIAM Journal on Computing 39(3), 1153-1218 (2009)

[5] Haitner, I., Reingold, O., Vadhan, S., Wee, H.: Inaccessible entropy. In: Proceedings of the 41st Annual ACM Symposium on Theory of Computing (STOC). ACM Press, New York (2009)

[6] Haitner, I., Reingold, O., Vadhan, S.: Efficiency improvements in constructions of pseudorandom generators. In: Proceedings of the 42th Annual ACM Symposium on Theory of Computing (STOC). ACM Press, New York (2010)

[7] Håstad, J., Impagliazzo, R., Levin, L.A., Luby, M.: A pseudorandom generator from any one-way function. SIAM Journal on Computing 28(4), 1364-1396 (1999); Preliminary versions in STOC 1989 and STOC 1990

[8] Impagliazzo, R., Luby, M.: One-way functions are essential for complexity based cryptography. In: Proceedings of the 30th Annual Symposium on Foundations of Computer Science (FOCS), pp. 230-235 (1989)

[9] Katz, J., Koo, C.: On constructing universal one-way hash functions from arbitrary one-way functions. Technical Report 2005/328, Cryptology ePrint Archive (2005)

[10] Naor, M., Yung, M.: Universal one-way hash functions and their cryptographic applications. In: Proceedings of the 21st Annual ACM Symposium on Theory of Computing (STOC), pp. 33-43. ACM Press, New York (1989)

[11] Rompel, J.: One-way functions are necessary and sufficient for secure signatures. In: Proceedings of the 22nd Annual ACM Symposium on Theory of Computing (STOC), pp. 387-394 (1990)

[12] Rompel, J.: Techniques for computing with low-independence randomness. PhD thesis, Massachusetts Institute of Technology (1990), http://dspace.mit.edu/handle/1721.1/7582

[13] Shoup, V.: A composition theorem for universal one-way hash functions. In: Preneel, B. (ed.) EUROCRYPT 2000. LNCS, vol. 1807, pp. 445-452. Springer, Heidelberg (2000) 